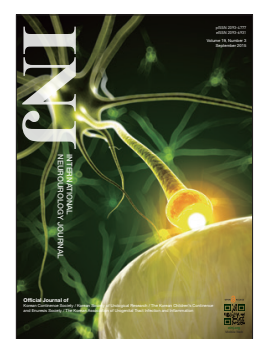

\title{
Interleukin-33 as a Potential Therapeutic Target for Interstitial Cystitis: New Perspectives on Mast Cell Regulation
}

\author{
Min Soo Choo \\ Department of Urology, Hallym University College of Medicine, Chuncheon, Korea \\ E-mail: snuhuro@gmail.com
}

There are many problems still unresolved by modern medicine. In the field of voiding disorders, interstitial cystitis (IC) remains a representative disease yet to be conquered. The exact pathophysiologic mechanism of IC is not understood. Although several hypotheses have been proposed, including leaky epithelium, mast cell activation, and neurogenic inflammation, no theory has been clearly established yet [1]. IC may have multiple causes and may represent a final common reaction of the bladder to multifactorial etiology. Most researchers believe that the mast cell plays a key role in the common pathophysiology of IC.

Despite many efforts to clarify the etiology of IC, our knowledge is insufficient for useful insights into effective treatments [2]. The current therapeutic goal is the improvement of quality of life, and not curative treatment. It is believed that histamine, which is secreted from mast cells, causes specific symptoms of IC such as pain, hyperemia, and fibrosis. Therefore, antihistamine treatment to adjust mast cell activation is considered a scientifically robust and rational therapy for IC. However, results using antihistamines in clinical practice were disappointing. Sufficient evidence does not exist to conclusively state that antihistamines effectively regulate the activation of mast cells in the bladder [3].

In this issue of the International Neurourology Journal, we have published an article reviewing new perspectives on mast cell regulation [4]. It was confirmed in other organs that interleukin (IL) 33 can modulate allergic inflammation. IL-33 is closely related to mast cell activation, which is considered the core of IC pathogenesis. Therefore, it may be possible to regulate mast cell activation by inhibiting IL-33. Promising results have been reported in a number of studies. However, because the reaction of the anti-IL33 could vary in different organs, we have many challenges to overcome before systemic administration of anti-IL-33 as a therapeutic agent can be implemented. Nevertheless, this is valuable work as it presents a potential therapeutic target for an incurable disease yet unconquered by modern medicine.

The most important message of this review for urologists is included in the last sentence of conclusion: "co-operative research among allergologists and urologists could yield more meaningful conclusions about the pathophysiologic mechanism and treatment strategies for interstitial cystitis" [4].

- Conflict of Interest: No potential conflict of interest relevant to this article is reported.

\section{REFERENCES}

1. Fiehn O, Kim J. Metabolomics insights into pathophysiological mechanisms of interstitial cystitis. Int Neurourol J 2014;18:106-14.

2. Lee T. New insights into the mechanism of the down-regulation of mast cells in the treatment of interstitial cystitis: possible role of Siglecs. Int Neurourol J 2011;15:59-60.

3. Fall M, Oberpenning F, Peeker R. Treatment of bladder pain syndrome/interstitial cystitis 2008: can we make evidence-based decisions? Eur Urol 2008;54:65-75.

4. Jang TY, Kim YH. Interleukin-33 and mast cells bridge innate and adaptive immunity: from the allergologist's perspective. Int Neurourol J 2015;19:142-50. 